\title{
ROLE OF THE SUPRACHIASMATIC NUCLEI IN THE CIRCADIAN SYSTEM OF THE HOUSE SPARROW, PASSER DOMESTICUS ${ }^{1}$
}

\author{
JOSEPH S. TAKAHASHI ${ }^{2}$ AND MICHAEL MENAKER ${ }^{3}$ \\ Institute of Neuroscience, University of Oregon, Eugene, Oregon 97403
}

Received December 15, 1981; Accepted February 5, 1982

\begin{abstract}
The suprachiasmatic nuclei (SCN) of the hypothalamus are necessary for the persistence of free running circadian activity rhythms in house sparrows. Suprachiasmatic lesions abolish circadian locomotor rhythms in constant darkness. The locomotor activity of lesioned sparrows was analyzed with two different power spectral analysis methods and was found to be arrhythmic. There was a weak correlation between the extent of damage to the SCN and the relative amplitude of the power spectral density in the circadian frequency range. In light-dark cycles (LD 12:12), the locomotor behavior of lesioned sparrows was rhythmic and similar to that of intact birds. However, entrainment was disrupted in SCN-lesioned sparrows exposed to a short photoperiod light cycle (LD 1:24).

These results demonstrate that the SCN are crucial for the generation of overt circadian rhythmicity in birds. The fact that SCN lesions abolish circadian rhythms in sparrows and several mammalian species suggests that vertebrate circadian organizalion may be based on differentially weighted interactions among the pineal, the SCN, and perhaps other brain regions.
\end{abstract}

The pineal gland plays a major role in the circadian organization of passerine birds (Takahashi and Menaker, 1979a). Removal of the pineal eliminates the persistence of free running locomotor rhythms (Gaston and Menaker, 1968; Gaston, 1971; McMillan, 1972), transplantation of pineal tissue restores rhythmicity in arrhythmic pinealectomized hosts (Zimmerman and Menaker, 1975; Menaker and Zimmerman, 1976), and the restored rhythm bears the phase of the donor (Zimmerman and Menaker, 1979). The reconstitution of free running rhythms and the transfer of phase information in transplant recipients provide the strongest evidence available that the pineal acts as a dominant pacemaker within the circadian system of the house sparrow.

'These experiments were performed in part at the Department of Zoology, University of Texas, Austin, TX 78712. The research was supporied hy National Institutes of Health Grants HD-03803 and HD07727 to M. M. and a National Science Foundation graduate fellowship and National Institute of General Medical Sciences National Research Award 5T32 GM 07257 in Systems and Integrative Biology to J. S. T. We thank R. Van Buskirk, G. Vaaler, G. Wyche, E. Kluth, D. Schaffer, and P. Lambert for technical assistance. We are grateful to Drs. Gwinner, Strumwasser, Pittendrigh, Aschoff, Eskin, Rusak, and Enright for their suggestions and stimulating ideas.

${ }^{2}$ Present address: Section on Pharmacology, Laboratory of Clinical Science, Building 10, Room 2D-47, National Institute of Mental Health, Bethesda, MD 20205.

${ }^{3}$ To whom reprint requests should be addressed at Institute of Neuroscience, 75 Science II, University of Oregon, Eugene, OR 97403.
Although the pineal is necessary for the persistence of rhythmicity in constant conditions, two observations clearly show that oscillatory components remain in pinealectomized sparrows. First, the locomotor activity of pinealectomized birds can be entrained to light-dark cycles. Second, there is a transient oscillation of the locomotor activity which decays and gradually approaches aperiodicity after transfer from a light cycle to constant darkness (Gaston and Menaker, 1968; Gaston, 1971). The synchronization of the rhythm to light cycles and the transient persistence of rhythmicity in constant conditions demonstrate that an entrainable component that behaves as a damped oscillator remains after pineal removal (however, see Takahashi and Menaker, 1982). These observations make it clear that structures other than the pineal are involved in the circadian system of sparrows. The identity of these components is unknown.

In mammals, the suprachiasmatic nuclei (SCN) of the hypothalamus are necessary for the maintenance of circadian rhythmicity (Moore, 1978; Rusak and Zucker, 1979). Lesions that destroy these nuclei abolish a variety of physiological and behavioral rhythms in several rodents (Moore and Eichler, 1972; Stephan and Zucker, 1972; Moore and Klein, 1974; Stetson and Watson-Whitmyre, 1976; Rusak, 1977; Ibuka et al., 1977; Brown-Grant and Raisman, 1977). Such lesions, which interrupt retinal input to the hypothalamus (Moore and Lenn, 1972; Hendrickson et al., 1972; Eichler and Moore, 1974), also severely disrupt entrainment to light-dark cycles in ro- 
dents. These results and especially those of Inouye and Kawamura (1979) and Schwartz et al. (1980) demonstrate that the SCN are crucial elements and suggest that the SCN may act as a pacemaker in the mammalian circadian system.

A homologue of the mammalian SCN has been described in the house sparrow, Passer domesticus (Crosby and Woodburne, 1940; Crosby and Showers, 1969). There is also evidence for a direct retinal projection to the SCN in $P$. domesticus (Hartwig, 1974) and other avian species (Meier, 1973; Bons, 1976). In view of the anatomical similarities of the SCN in birds and mammals, we speculated that functional similarities also might exist. Indeed, this appears to be correct. Our results demonstrate that the SCN of the house sparrow are necessary for the expression of endogenous circadian rhythmicity in constant darkness. Since the pineal organ is known to play a dominant role in the circadian system of this species, our new results have important implications concerning the organization of avian circadian systems.

\section{Materials and Methods}

\section{Animals}

House sparrows (Passer domesticus) of both sexes were trapped in the vicinity of Austin, TX and held in outdoor aviaries until use. The birds were given fresh water and a mixture of millet, Purina Layena, and grit.

\section{Locomotor recording}

Experimental birds were housed singly in light-tight wooden boxes. Remotely controlled fluorescent lamps (4W cool white, Ken-Rad P4T5) in the top of each box provided a light intensity of approximately 50 lux on the cage floor. Because sparrows are susceptible to social entrainment by sound (Menaker and Eskin, 1966), the recording boxes were acoustically isolated from each other by providing the room with a constant background of random noise of $92 \mathrm{~dB}$. Food (millet, Purina Layena, and grit) and water were available continuously.

Each box contained a double breeder bird cage equipped with two perches attached to microswitches wired in parallel. The switches from each recording cage were connected to an interface that was activated by a switch transition. The quantity of 10-sec intervals during which more than one switch transition occurred was counted and summed every $10 \mathrm{~min}$ by a Control Data 160-A computer. For each 10 -min bin, there was a maximum of 6010 -sec intervals during which activity could have occurred, yielding a high frequency cutoff for the collection system of 0.1 event $\mathrm{sec}^{-1}$. The counts for each 10-min bin were collected for 216 locomotor recording channels and punched on paper tape. Data recorded on paper tape were subsequently transferred to magnetic tape from which they could be retrieved and analyzed by computer. For visual display, time series of locomotor data were computer-plotted to simulate Esterline Angus event recorder actographs.

\section{Stereotaxic procedures}

Sparrows were anesthetized with a solution of sodium pentobarbital (1:5 dilution of Nembutal with sterile water) injected into the pectoral muscle at a dose of $40 \mathrm{mg} /$ kg. Birds were placed into a David Kopf 900 stereotaxic instrument equipped with rat ear bars and a mouse incisor bar. The ear bars were inserted into the external auditory canals and the beak was held at an angle $30^{\circ}$ below horizontal (beak bar position: anterior, $16.8 \mathrm{~mm}$; ventral, $9.8 \mathrm{~mm}$ from ear bar zero). The feathers from the top of the head were plucked and a midsagittal incision was made to expose the skull. The electrode position was marked and a small hole was drilled through the skull exposing the midsagittal sinus. The middle of the sinus was used as the zero point for the lateral stereotaxic dimension. The dura was pierced on either side of the sinus and the electrode was lowered slowly to the desired depth. Coordinates for the SCN determined relative to the ear bars were: anterior, $2.1 \mathrm{~mm}$; lateral, $\pm 0.5 \mathrm{~mm}$; and dorsal, $3.3 \mathrm{~mm}$. Because the SCN of the sparrow are approximately $1.0 \mathrm{~mm}$ apart, it was necessary to make bilateral lesions in order to destroy both nuclei. Lesions were made with a Grass LM-3 lesion maker using a No. 1 stainless steel insect pin coated with Insl-X with about $0.5 \mathrm{~mm}$ of the tip exposed. Radiofrequency current (25 to $35 \mathrm{~mA}$ ) was passed for 30 to $60 \mathrm{sec}$. For sham operations, the electrode was lowered, but no current was applied. The electrode then was withdrawn and a similar lesion was placed on the contralateral side. A piece of Gelfoam was placed into the hole and the scalp was sutured together. After recovery from anesthesia, birds were returned to their experimental cages immediately.

In later experiments (see "DD lesion group"), a David Kopf 1730 stereotaxic instrument with rat ear bars and a pigeon beak bar was used. The beak was held at an angle $45^{\circ}$ below horizontal (anterior, $8.5 \mathrm{~mm}$; ventral, 8.5 $\mathrm{mm}$ from ear bar zero). Coordinates for the SCN determined relative to the ear bars were: anterior, $3.4 \mathrm{~mm}$; lateral, $\pm 0.5 \mathrm{~mm}$; and dorsal, $2.6 \mathrm{~mm}$. Radiofrequency lesions were made with a Grass LM-3 lesion maker using a Rhodes Medical Instruments electrode (RNEX-300). Current (25 to $35 \mathrm{~mA}$ ) was applied for at least $60 \mathrm{sec}$. Other surgical procedures were the same as those described above.

\section{Histology}

At the end of each experiment, the birds were anesthetized with Nembutal $(80 \mathrm{mg} / \mathrm{kg})$ and perfused transcardially with $0.75 \%$ saline and $10 \%$ neutral buffered formalin. The brain was removed and fixed in $10 \%$ formalin for at least 3 days. After fixation, the brains were transferred to $25 \%$ sucrose in $10 \%$ formalin solution. Tissue then was frozen and embedded in Lipshaw M-1 embedding matrix and sectioned in a cryostat or was embedded in egg yolk and gelatin for sectioning. Frozen sections (25 or $40 \mu \mathrm{m}$ thick) were cut serially in the frontal plane through the anterior hypothalamus and the entire lesion site. Brain sections were stained with cresyl violet and Luxul fast blue $G$ (Kluver and Barrera, 1953) and examined to determine the location and extent of the lesions. Drawings were made of each lesion site and the extent of damage to the SCN and adjacent regions was estimated.

\section{Power spectral analysis}

Continuous time series of quantitative locomotor data were analyzed for rhythmicity by discrete Fourier transformation. Two different methods were used to calculate 
the power spectrum: (1) computation of the power spectral density function from the autocovariance function (Blackman and Tukey, 1958) and (2) direct computation of the power spectral density function using the fast Fourier transform (Cooley and Tukey, 1965).

Blackman and Tukey method. FORTRAN programs were written and used to analyze digital locomotor data on a Control Data Cyber 170 computer. Record lengths of 20 to 30 days composed of $10-\mathrm{min}$ sample intervals (2880 to 4320 points) were selected for each bird. Each time series was normalized to a mean value of 0.0 and a variance of 1.0. The record was divided into 12 equal intervals and the mean square value was calculated for each interval. Stationarity of the record was tested by a nonparametric "run" test upon the sequence of the 12 mean square values using a 0.05 level of significance (Bendat and Piersol, 1971). The data were "pre-whitened" using Tukey's method (Blackman and Tukey, 1958, p. 52). The autocovariance function was computed for 433 lag values. Raw estimates of the power spectral density function were calculated for 433 equally spaced, overlapping values using trapezoidal integration. A Hanning window was applied as a convolution in the frequency domain to obtain smoothed estimates of the power spectral density. The smoothed estimates then were "formed" or "postdarkened" to yield the final estimates of the power spectral density function. This yielded a spectral bandwidth of $0.0139 \mathrm{hr}^{-1}$ and 20 degrees of freedom. Using the Blackman and Tukey method, the integral of the power spectral density function should equal two times the sample variance (there is a factor of 2 because the spectral estimates overlap and therefore are redundant). Because the total variance of the original time series was normalized to a value of 1.0 , this had the effect of normalizing the total power of the spectral density function. The variance calculated by integrating the power spectral density function was uniform across all records tested.

Fast Fourier transform method. The records used above also were analyzed by direct computation of the fast Fourier transform using the Cooley-Tukey method (Cooley and Tukey, 1965). Programs written in FORTRAN were used to perform the analyses on a Control Data 6600 computer. Record lengths were adjusted to a value of 4096 points. Long records were truncated and short records were padded with zeros. A probability density function of the raw data was determined. The sample mean was adjusted to a value of 0.0 and the variance was adjusted to 1.0. Stationarity of the record was tested by applying the "run" test to the mean square values calculated for 16 equal subdivisions of the record. A "four-sample Blackman-Harris" window was applied as a product in the time domain as recommended by Harris (1978). Using a subroutine written by Otnes, Nathans, and Enochson (Otnes and Enochson, 1978), the fast Fourier transform was calculated. Raw power spectral density estimates were computed for 2049 points. These estimates were adjusted for the power loss due to tapering the data with the window. The scale factor (1/0.28967) was determined by integrating the square of the window function. Smoothed estimates of the power spectral density then were obtained by frequency-averaging 10 contiguous raw estimates. This method yielded a spectral bandwidth of $0.01465 \mathrm{hr}^{-1}$ and 20 degrees of freedom.

\section{Validation of the spectral analysis methods}

The amplitude of the power spectrum is correlated with the level of the sample data. Because surgical procedures commonly reduce the level of locomotor activity expressed by birds, a reduction in power of a peak in the circadian frequency range can result. In order to minimize the confounding effects of the activity level upon the amplitude of a circadian frequency component, we chose to normalize the total power of the power spectrum. For a given set of parameters, the power spectral density at a particular frequency represents the proportion of the total power (or variance) expressed at that frequency. Because the integral of the power spectral density function is equal to the sample variance, the power spectra obtained from both methods described above should have been normalized as a result of adjusting the sample variance to a value of 1.0. This assumption was tested by computing spectra for artificial data composed of sine waves, pseudorandom number series, or a combination of a sine wave mixed with various amplitudes of pseudorandom noise.

The power spectral density of a sine wave with a 24 -hr period containing pseudorandom noise of known variance was correlated directly with the proportion of the total variance that was contributed by the sine wave (Fig. 1). The value of the power spectral density did not vary as a function of the level of the data except at very low average sample values (less than 1.0 event/10-min interval). This distortion was probably due to the digital nature of the data. The Blackman and Tukey method was less sensitive to low sample mean values than the fast Fourier transform method. This improved performance probably resulted from the sample autocovariance function being less sensitive to the effects of digitizing than the fast Fourier transformation. The Blackman and Tukey method resulted in more appropriate frequency values for circadian data than the fast Fourier transform method. However, if Singleton's (1969) mixed radix fast Fourier transform algorithm was used, then appropriate frequency values could be obtained. Computational speed was, of course, faster with the fast Fourier transform.

\section{Experimental treatments}

Five groups of sparrows were examined.

Unoperated group. These birds were held on LD 12:12 for at least 1 week and then transferred to constant darkness for 3 months ( $N=10 ; 10$ with spectral analysis).

Sham group. These birds were held on LD 12:12 for 9 weeks, sham operated, transferred to constant darkness 2 weeks later, and maintained in constant darkness for 8 weeks ( $N=5 ; 4$ with histology and spectral analysis).

LD 12:12 lesion group. These birds were held on LD 12:12 for 2 to 12 weeks, lesioned, transferred to constant darkness 2 to 8 weeks later, and maintained in constant darkness for at least 4 weeks ( $N=47 ; 30$ with histology and spectral analysis).

LD 6:18 lesion group. These birds were held on LD $6: 18$ for at least 3 weeks, lesioned, transferred to constant darkness 2 weeks later, and maintained on constant 

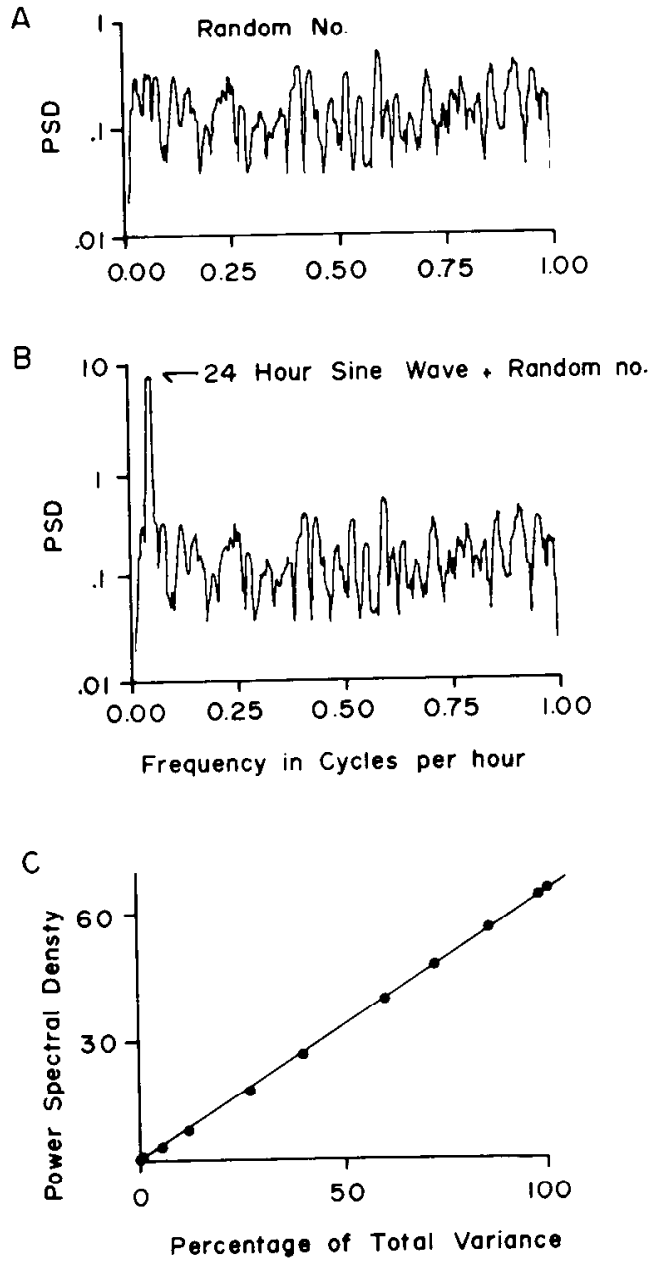

Figure 1. Power spectral analysis. A, Power spectral density $(P S D)$ from a series of computer-generated random numbers using the fast Fourier transform method; $B$, power spectral density from a series of random numbers with a 24-hr sine wave added; $C$, the relationship between the power spectral density and the percentage of the total variance contributed by a $24-\mathrm{hr}$ sine wave.

darkness for at least 4 weeks $(N=6 ; 3$ with histology and spectral analysis).

DD lesion group. Female birds were held on LD 6:18, transferred to constant darkness for 20 to 29 days, lesioned, and maintained in constant darkness for at least 4 weeks ( $N=17 ; 6$ with histology and spectral analysis).

Although the procedural details in the three lesioned groups were different, their major results were comparable and will be reported together.

\section{Results}

Hypothalamic lesions were made in 70 sparrows whose locomotor behavior was recorded in constant darkness. Of these, 59 birds were examined histologically, and 44 birds generated activity records in constant darkness that were suitable for spectral analysis. A total of 39 lesioned sparrows were examined in both ways.

Figure 2 shows the appearance of the SCN of the house sparrow. These nuclei are located bilaterally on either side of the ventral portion of the third ventricle immediately above the rostral portion of the optic chiasm.
Each nucleus is oval in cross-section and appears laminated. In the sparrow, the borders of the nuclei are diffuse and are more difficult to distinguish than are those of mammalian nuclei.

The general location of the lesions is diagramed in Figure 3. Lesions were restricted to the medial ventral portions of the anterior hypothalamus. All lesions were caudal to the rostral border of the optic chiasm, medial to the lateral division of the supraoptic nucleus, and rostral to the ventral supraoptic decussation. Most lesions lay in the region of the suprachiasmatic nuclei and the dorsal supraoptic decussation. Variable damage was found in the preoptic periventricular nucleus, medial preoptic area, anterior hypothalamic area, and optic chiasm.

Effects of sham operations. Sham operations had no effects on the locomotor behavior of sparrows. Shamoperated birds expressed clear free running rhythms in constant darkness. Spectral analysis of their records showed strong peaks at circadian frequencies and minor peaks at harmonic frequencies (Fig. 4). Both the amplitude of the power spectral density corresponding to circadian frequencies and the activity level of sham-operated sparrows were indistinguishable from estimates for intact controls (Table 1).

Behavior of SCN-lesioned sparrows in constant darkness. Lesions that destroyed at least $90 \%$ of both SCN severely disrupted free running locomotor rhythms of sparrows held in constant darkness. Examples are shown in Figures $5 A$ and $6 A$. No circadian and few ultradian components were detected in the power spectra from SCN-lesioned sparrows. Partial or unilateral SCN lesions did not abolish circadian rhythmicity. In some cases, the precision of the rhythm was reduced (Figs. $5 B$ and $6 B$ ) or the free running period was changed (Fig. $6, C, D$, and $E)$. In birds with large but incomplete SCN lesions, circadian rhythmicity alternated with transient intervals of apparent arrhythmicity (Fig. 7, $A$ and $B$ ). In some of these records, there was weak evidence for components of the locomotor activity which appeared to dissociate and free-run.

Table I summarizes the results of the power spectral analysis of the activity records of sparrows maintained in constant darkness. Results using the Blackman and Tukey method and the fast Fourier transform method are shown. Large SCN lesions severely reduced the power spectral density at circadian frequencies. Medium and small SCN lesions reduced the amplitude of the circadian peak but did not abolish it. Some of the reduction in power appears to be nonspecific because birds with lesions causing no SCN damage also had reduced power in the circadian peak. There was a weak, but significant, correlation between the extent of damage to the SCN and the amplitude of the power spectral density in the circadian range (Table I).

Hypothalamic lesions reduced the level of locomotor activity. This reduction was not due to general surgical manipulations because sham-operated birds had normal activity levels. All lesion groups expressed lower activity than the controls. The group with large SCN lesions had the lowest levels of activity. Because the spectral analysis methods normalized the variance of the time series of 

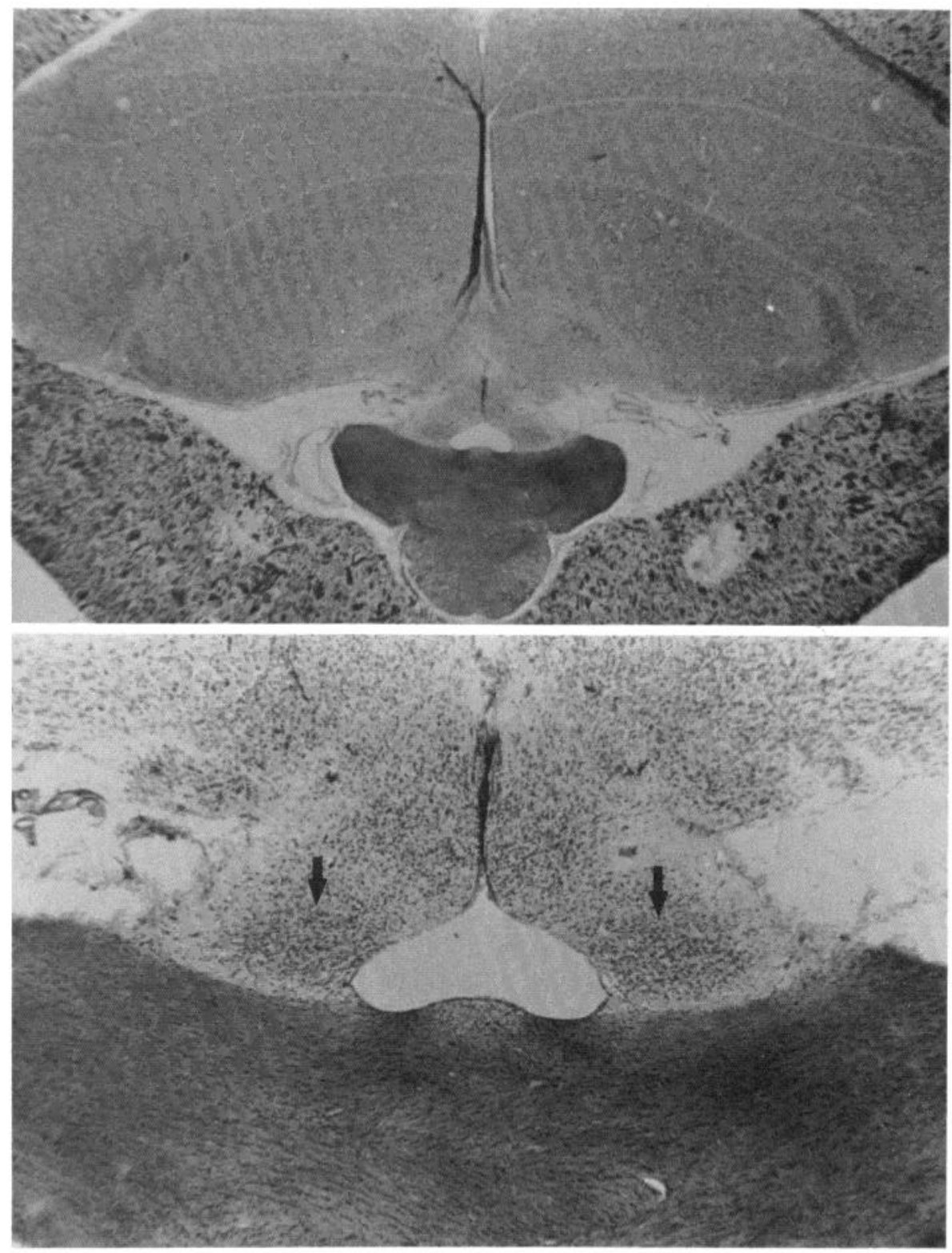

Figure 2. Histological sections showing the location of the suprachiasmatic nuclei in the house sparrow. Frontal sections ( $25 \mu \mathrm{m}$ thick) stained with Luxol fast blue and cresyl violet are shown. Top, Frontal section through the forebrain; bottom, magnification of chiasmatic area. The arrows indicate the suprachiasmatic nuclei.

locomotor activity, the effects of reductions in activity level upon the total power of the spectra were compensated. However, the digital nature of the data caused some power loss at very low activity levels. Because the average level of activity expressed by sparrows in constant darkness was low, this undoubtedly affected the reliability of the analysis. The Blackman and Tukey method was least sensitive to the effects of digitizing and was therefore the preferred method to analyze these data.

Although there was general agreement between the two different methods of calculating the power spectral density function, there were striking differences in the detailed form of the spectra. These included differences in the amplitude of coherent peaks and in the contribution of harmonic frequencies. Differences in the window

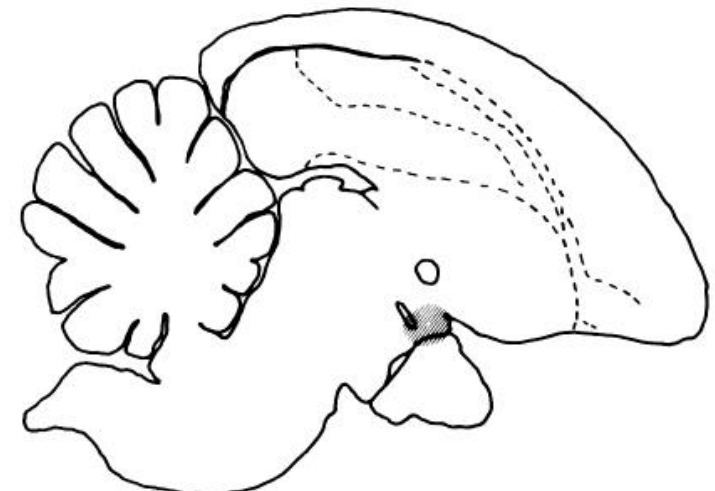

Figure 3. Diagram showing the general location of hypothalamic lesions (hatched area) in the house sparrow (midsagittal section). 


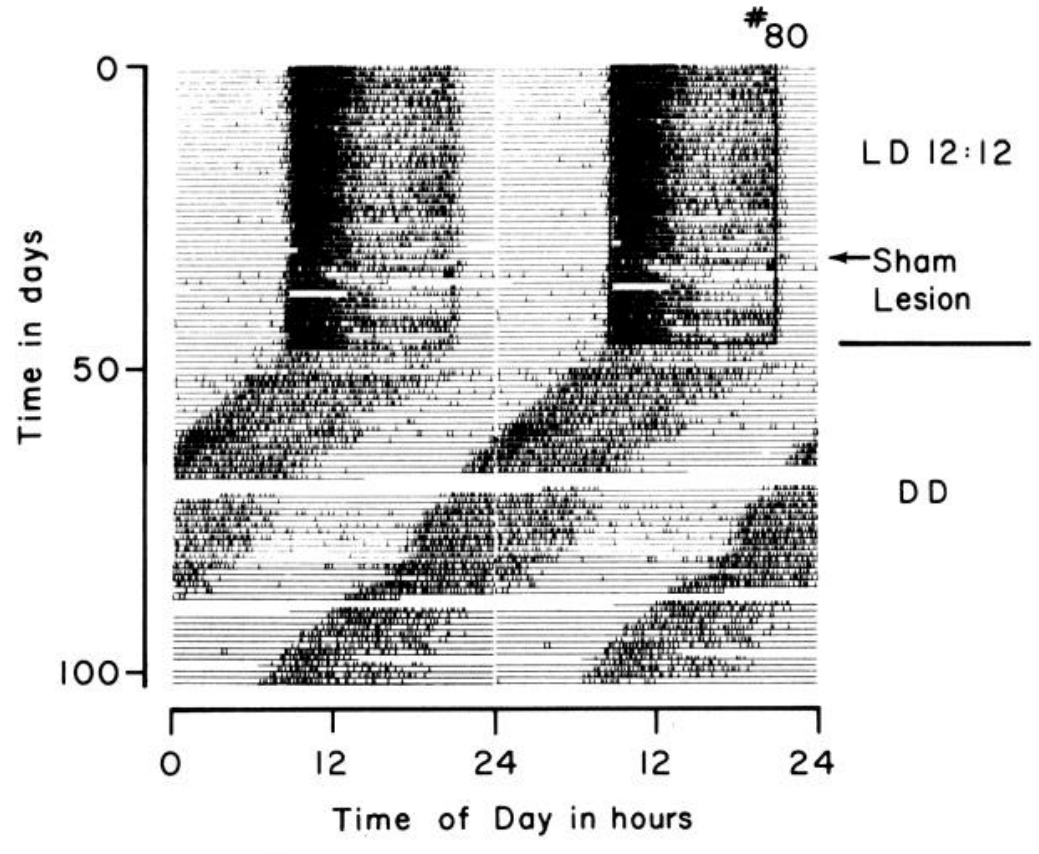

Figure 4. Top, Locomotor record from a shamlesioned sparrow; bottom, power spectra from bird 80 on days 49 to $69 . B T$, Power spectrum obtained using the Blackman and Tukey method; $D D$, total darkness; FFT, power spectrum obtained using the fast Fourier transform method; $P S D$, power spectral density. The arrows indicate the $24-\mathrm{hr}$ component.

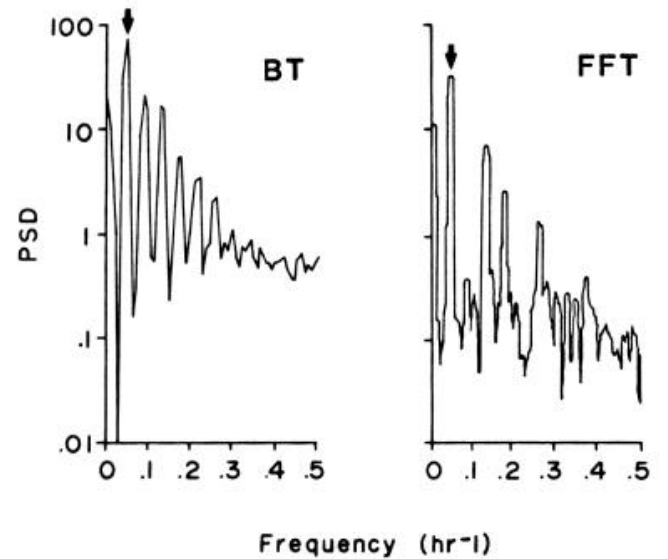

function, the Fourier transform algorithm, and the smoothing procedure were probably responsible for these changes in the power spectra. These analyses clearly show that the details of the power spectra obtained from Fourier analyses depend to a large extent upon the specific methods used. This variability should be kept in mind when interpreting the results of such analysis methods.

Effects of SCN lesions on entrainment. When sparrows that were arrhythmic as a result of SCN lesions were exposed to light-dark cycles, they expressed rhythmic activity patterns which were similar to the entrainment patterns of unoperated birds (Fig. 5A). All lesioned sparrows entrained normally on LD 12:12. Since activity coincided with the light portion in LD 12:12, it was not clear whether the rhythmic pattern was a result of activity directly forced by the light (masking) or entrainment of an oscillator. To examine this further, sparrows were exposed to an LD 1:24 light cycle chosen because intact sparrows reliably entrain to it with a stable phase lead of the activity rhythm. Since the light portion is short, this light cycle is probably a weaker entraining stimulus than LD 12:12.
Sparrows that were completely arrhythmic in constant darkness did not entrain normally to the LD 1:24 cycle (Fig. 6A). Sparrows that showed weak circadian components in constant darkness or that were only transiently arrhythmic entrained normally (Fig. 7, $A$ and $B$ ). The disruption of entrainment in lesioned sparrows on LD $1: 24$ is consistent with what is known concerning the involvement of both retinal and extraretinal photoreceptors in mediating entrainment information in this species (Menaker and Underwood, 1976). Because retinal and extraretinal photoreceptors are additive in their contribution to entrainment, SCN lesions would be expected to reduce the total photic input for entrainment by reducing retinal input mediated by the retinohypothalamic tract.

\section{Discussion}

While it is well established that the mammalian SCN are important in the regulation of circadian phenomena (Moore, 1978; Rusak and Zucker, 1979), there are few studies of the role of this region in birds. Lesions or cuts in the anterior hypothalamus have been shown to abolish several physiological rhythms in avian species. Ralph 

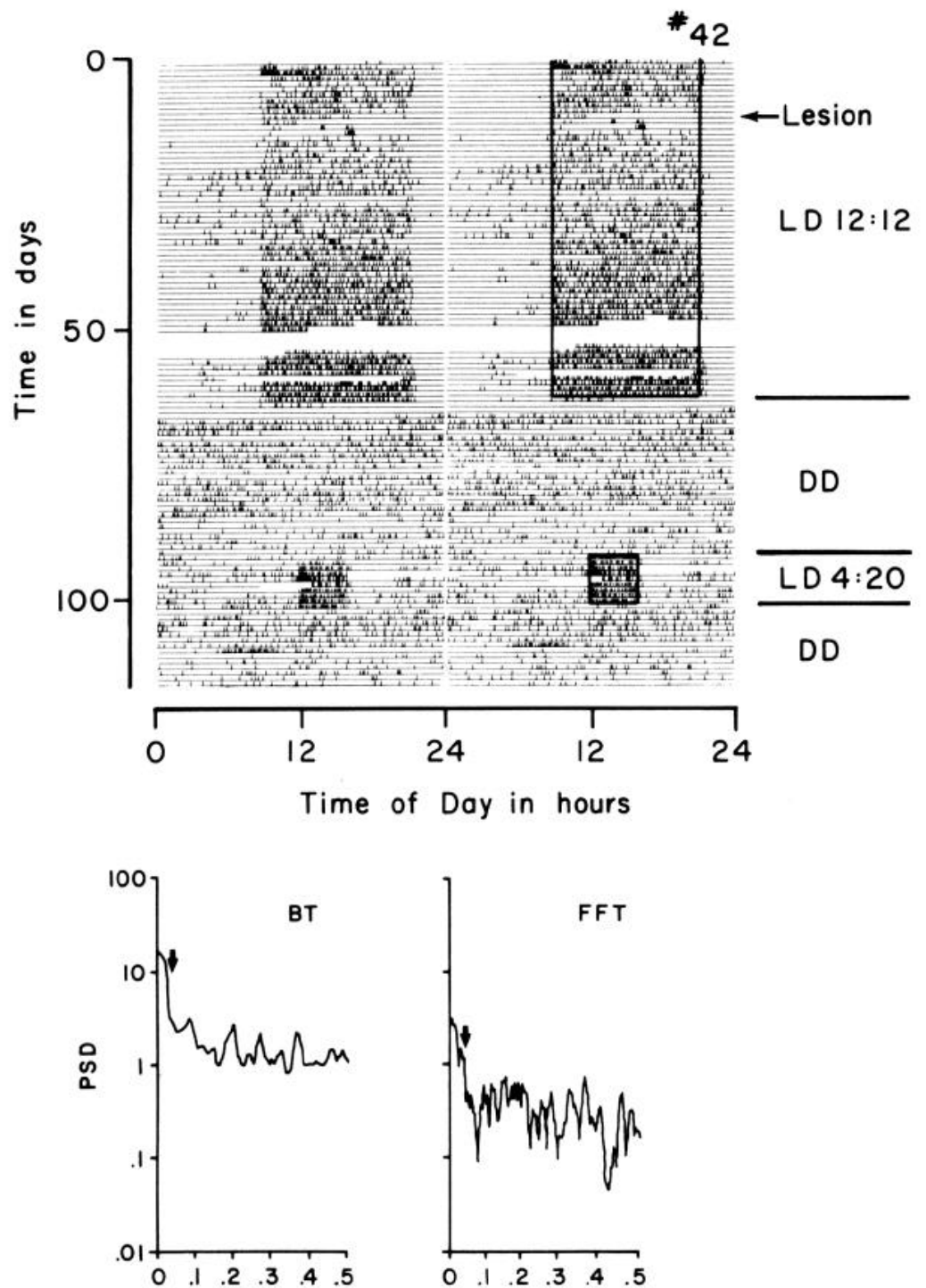

Figure 5. A: Top, locomotor record from a sparrow that received a lesion completely destroying the suprachiasmatic nuclei and the dorsal supraoptic decussation; bottom, power spectra from bird 42 on days 66 to 94 . $B$, Locomotor record from a sparrow bearing a lesion that destroyed only $10 \%$ of the SCN but caused extensive damage to the region posterior to the $\mathrm{SCN}$ including the dorsal supraoptic decussation. Conventions are as in Figure 4.

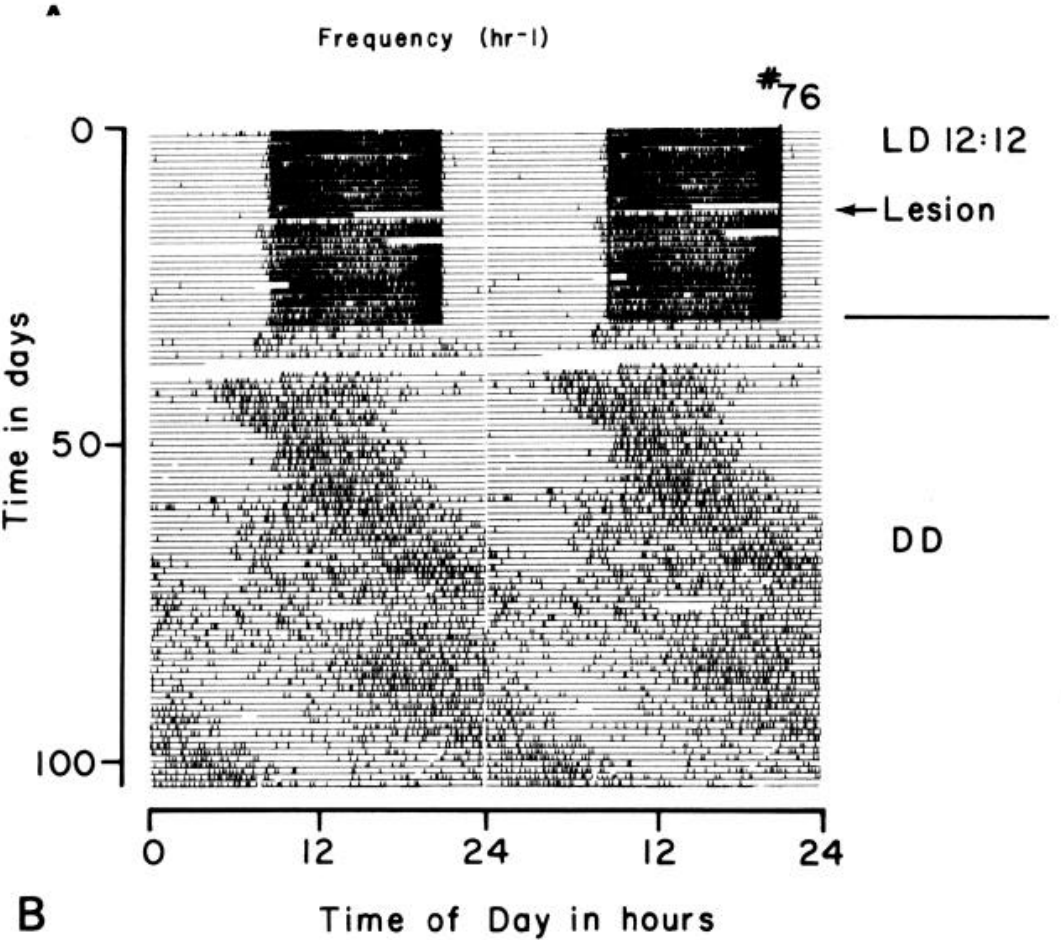



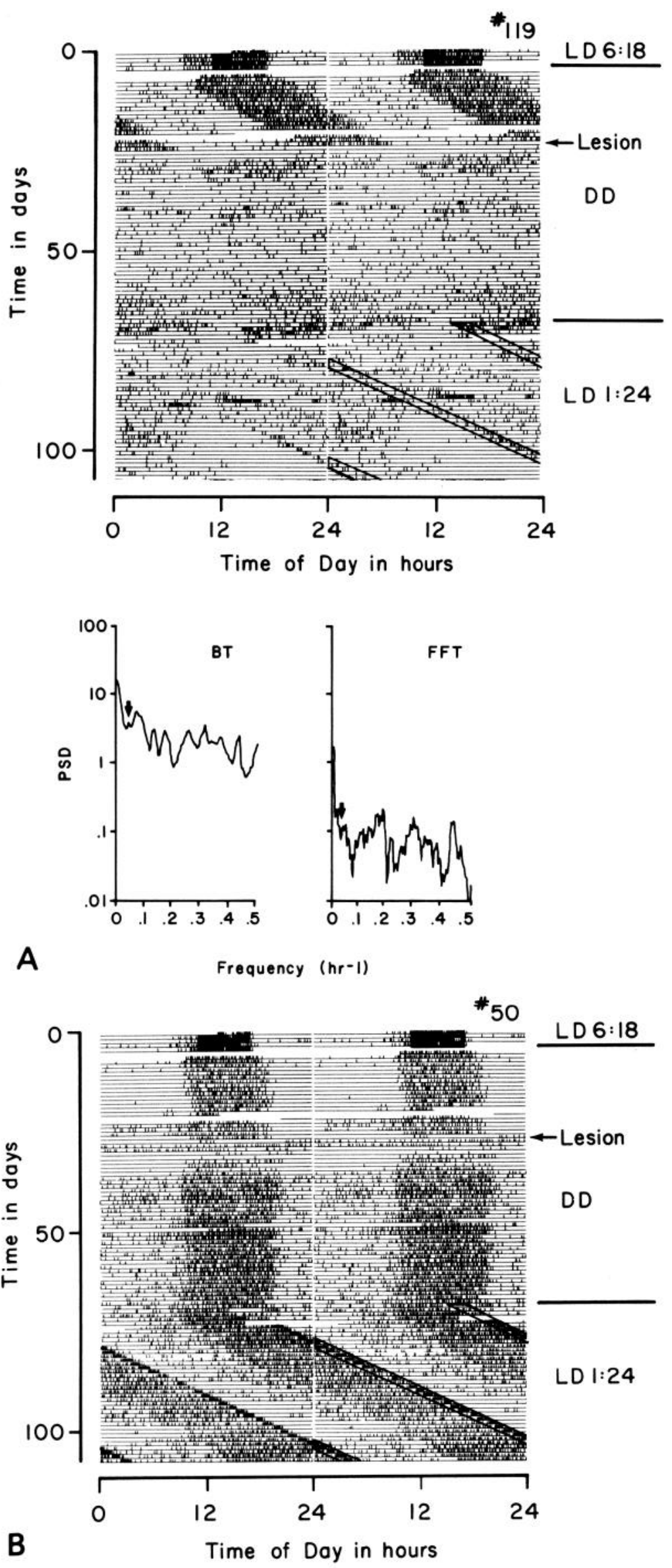

Figure 6. A: Top, locomotor record of a sparrow that received a lesion destroying 90 to $100 \%$ of the SCN and portions of the dorsal supraoptic decussation; bottom, power spectra from bird 119 on days 37 to 67 . $B$, Locomotor record from a sparrow receiving a lesion that destroyed about $35 \%$ of the SCN (most damage was unilateral and anterior). 


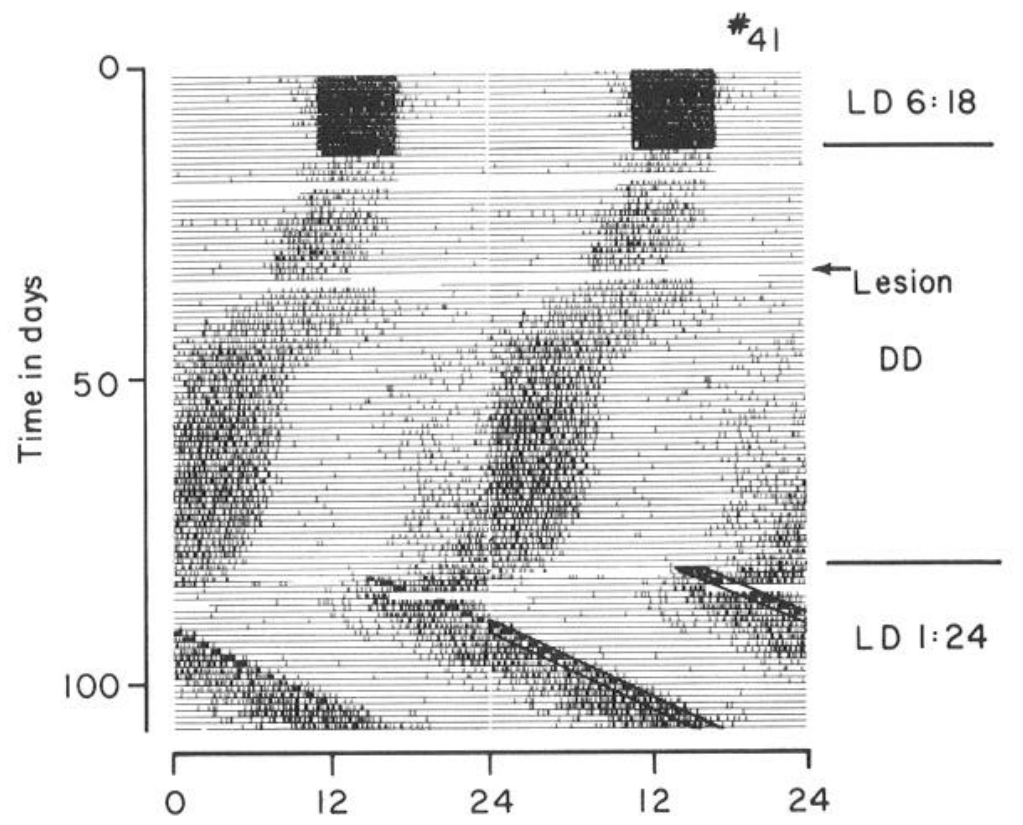

C Time of Day in hours

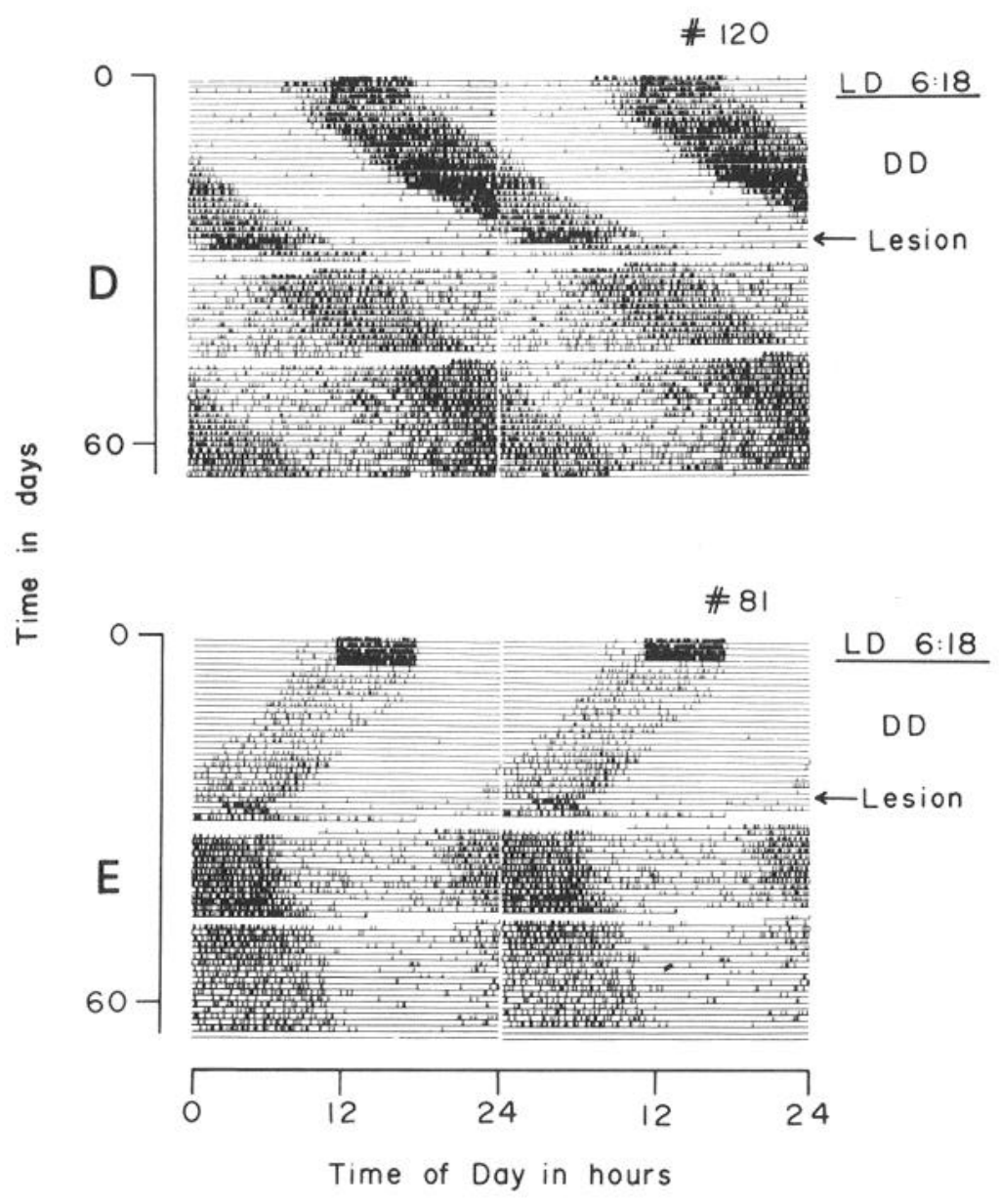

Figure 6. Continued. C, Locomotor record from a sparrow receiving a lesion that destroyed only about $20 \%$ of the ventral portion of the SCN and $100 \%$ of the dorsal supraoptic decussation. $D$, Record from a sparrow receiving a lesion destroying about $30 \%$ of the SCN (unilateral) with minimal damage to other areas. $E$, Record from a sparrow with a unilateral SCN lesion with minimal damage to other hypothalamic areas. Conventions are as in Figure 4.

(1959) has shown that such lesions in the hen prevent ovulation, which occurs at approximately $25 \mathrm{hr}$ intervals. Davies (1980) has clearly demonstrated that lesions of the supraoptic region of the Japanese quail interfere with ovulation if the lesions destroy the SCN as well as the supraoptic nuclei. Bouillé et al. $(1973,1975)$ have shown that hypothalamic deafferentation abolishes the diurnal plasma corticosterone rhythm in pigeons. Recently, Simpson and Follett (1980) have shown that anterior hypothalamic lesions abolish circadian locomotor activ- 


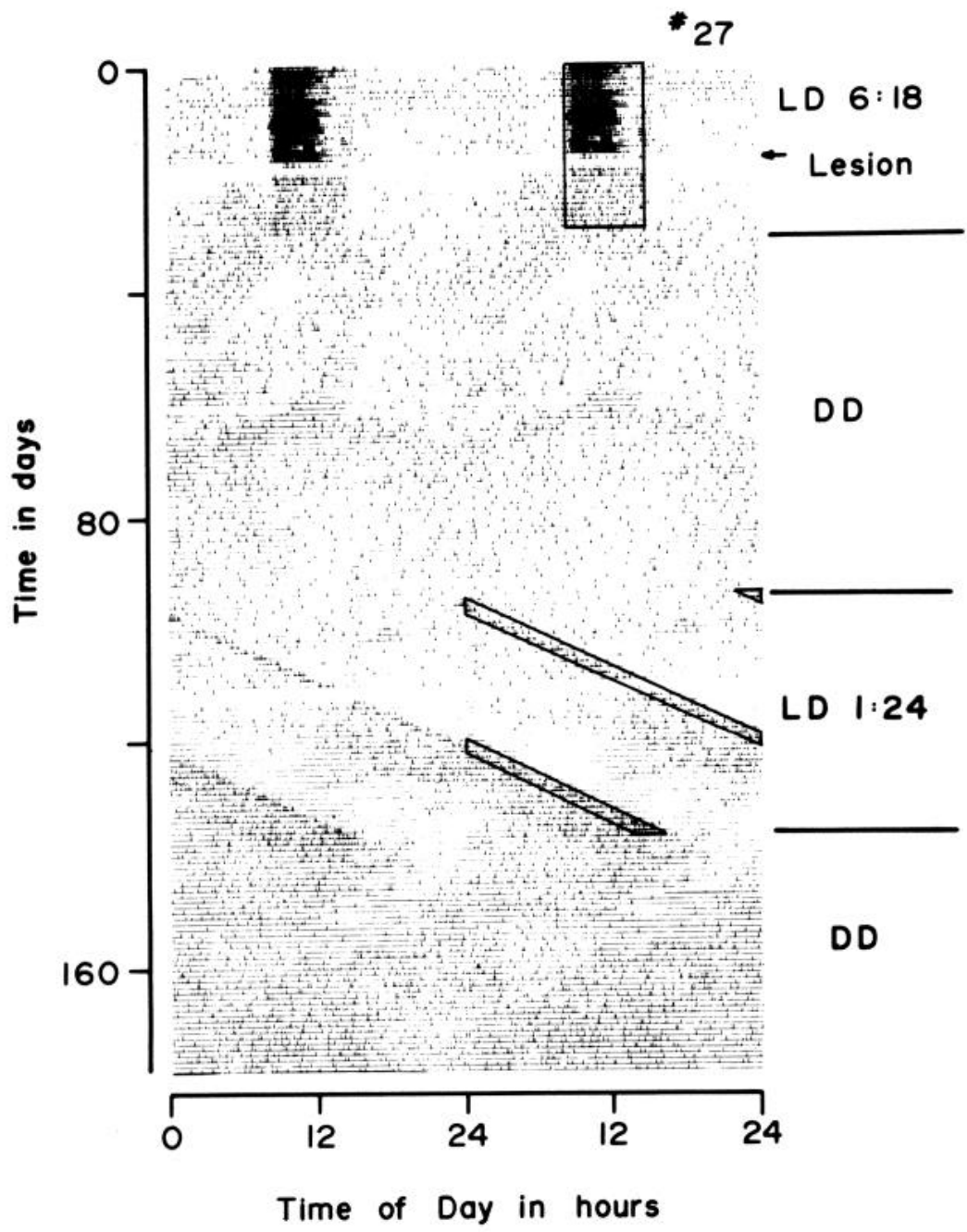

Figure 7. A: Top, locomotor record from a sparrow bearing a lesion that destroyed almost $90 \%$ of the SCN and $100 \%$ of the supraoptic decussation; bottom, power spectra from bird 27 on days 51 to 81.
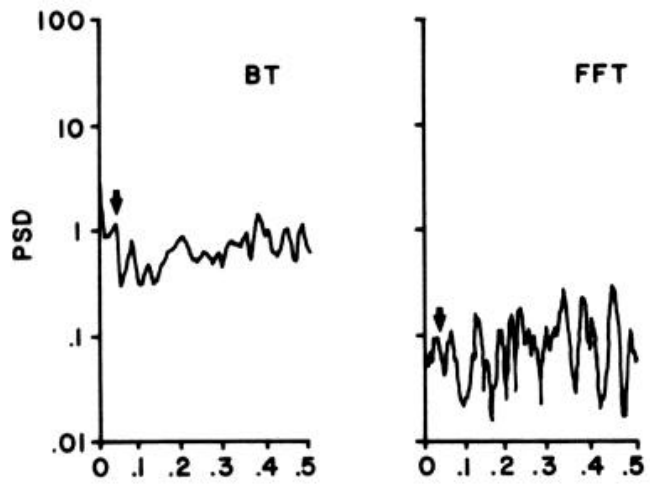

A

Frequency $(h r-1)$

ity rhythms in Japanese quail. Using a passerine species, the Java sparrow (Padda oryzivora), Ebihara and Kawamura (1981) now have confirmed our initial report (Takahashi and Menaker, 1979b) that SCN lesions abolish circadian rhythmicity. In all three species that have been examined (house sparrow, Java sparrow, and Japanese quail), the suprachiasmatic region appears critical for the maintenance of normal circadian rhythmicity. Clearly there are similarities in the neural control of circadian rhythms in birds and mammals. There are also

striking differences. Photoreceptive inputs to the circadian system of birds are numerous and varied (Menaker and Underwood, 1976). Both retinal and extraretinal photoreceptors influence the avian circadian system, whereas in mammals, only retinal photoreceptors are involved (Menaker et al., 1978; Rusak and Zucker, 1979). The pineal plays a central role in the control of circadian rhythms in passerine birds (Takahashi and Menaker, 1979a); while the pineal of mammals plays only a minor role, if any (Rusak and Zucker, 1979). The similarities 


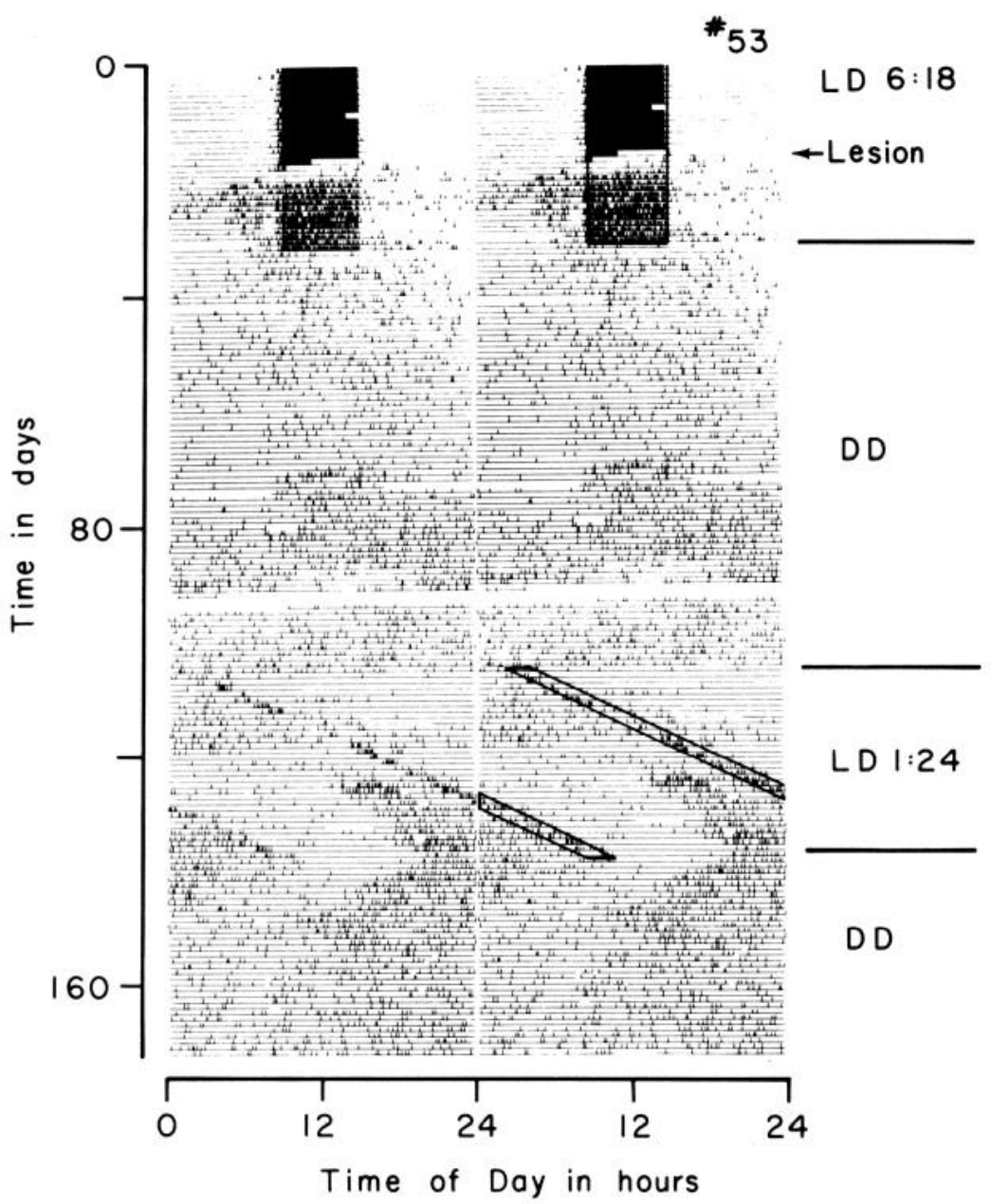

Figure 7. Continued. B: Top, locomotor record from a sparrow bearing a lesion that destroyed $60 \%$ of the posterior portion of the SCN and $100 \%$ of the supraoptic decussation; bottom, power spectra from bird 53 on days 44 to 74 . Conventions are as in Figure 4.
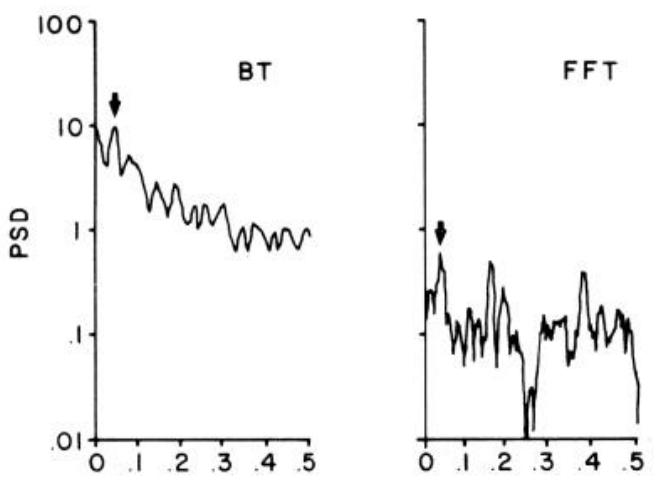

B

and differences between birds and mammals suggest that, although homologies exist, there is, yet to be discovered, a rich variety in the pattern of organization of circadian systems among the vertebrates.

The SCN of house sparrows play a crucial role in the regulation of circadian rhythmicity at the organismal level. They are necessary for the persistence of locomotor rhythms in constant darkness and are involved in the entrainment of activity rhythms to light cycles. Spectral analysis of the locomotor records revealed that circadian periodicities were absent and that few ultradian periodicities were present in lesioned sparrows. Although spar- rows bearing $\mathrm{SCN}$ lesions synchronized normally to LD 12:12 light cycles, they did not entrain to a relatively weak entraining agent (LD 1:24).

To a first approximation, the effects of SCN lesions are similar to those of pinealectomy in the sparrow. Both surgical procedures abolish free running rhythmicity in constant darkness but do not abolish synchronization to light-dark cycles. In the case of SCN lesions, however, we did not observe unequivocal cases of entrainment in sparrows that were completely arrhythmic. In LD 12:12 light cycles, it was difficult to observe a phase lead of the activity rhythm to the light cycle; and in LD 1:24, the 
TABLE I

Effect of hypothalamic lesions on circadian activity of house sparrows in constant darkness

\begin{tabular}{|c|c|c|c|c|c|}
\hline \multirow{2}{*}{ Group } & \multirow{2}{*}{$N$} & \multicolumn{2}{|c|}{$\mathrm{PSD}^{a}$} & \multirow{2}{*}{ Activity Level } & \multirow{2}{*}{$\Delta$ Body Weight } \\
\hline & & BT & $\mathrm{FFT}$ & & \\
\hline & & & & events $/ 10 \mathrm{~min}$ & $g m$ \\
\hline Intact & 10 & $\begin{array}{c}64.50 \pm 8.75 \\
(10 / 10)^{b}\end{array}$ & $\begin{array}{c}15.40 \pm 3.66 \\
(10 / 10)\end{array}$ & $2.90 \pm 0.617$ & \\
\hline Sham-operated & 4 & $\begin{array}{c}55.92 \pm 5.56 \\
(4 / 4)\end{array}$ & $\begin{array}{c}20.73 \pm 7.06 \\
(4 / 4)\end{array}$ & $2.81 \pm 0.878$ & $-0.575 \pm 0.813$ \\
\hline \multicolumn{6}{|l|}{ Lesions } \\
\hline \multicolumn{6}{|l|}{ Suprachiasmatic nuclei } \\
\hline Large $(90 \%)$ & 4 & $\begin{array}{c}2.23 \pm 0.57 \\
(0 / 4)\end{array}$ & $\begin{array}{c}0.286 \pm 0.116 \\
(0 / 4)\end{array}$ & $0.313 \pm 0.114$ & $-0.73 \pm 2.935$ \\
\hline Medium (25-90\%) & 15 & $\begin{array}{c}34.21 \pm 6.94 \\
(14 / 15)\end{array}$ & $\begin{array}{c}4.59 \pm 1.44 \\
(11 / 15)\end{array}$ & $1.00 \pm 0.243$ & $0.00 \pm 0.634$ \\
\hline
\end{tabular}

"Amplitude of the power spectral density (PSD) at circadian frequencies. Blackman and Tukey (BT): frequency $=0.04167 \mathrm{hr}^{-1}$; fast Fourier transform (FFT): frequency $-0.04175 \mathrm{hr}^{-1}$. Using a spectral bandwidth of $0.0139 \mathrm{hr}^{-1}$, the peak corresponds to frequencies ranging from 0.0347 to $0.0486 \mathrm{hr}^{-1}$ or period lengths from 28.8 to $20.6 \mathrm{hr}$, respectively. Pearson's correlation coefficient: BT: $r=-0.45 ; d f=51 ; p<0.001 ; \mathrm{FFT}$; $r=$ $0.41 ; d f=51 ; p<0.01$.

"The fraction of birds with a significant circadian peak.

entraining cycle may have been too weak to synchronize the system after lesioning. In sparrows, there are multiple photoreceptive inputs to the circadian system (McMillan et al., 1975). Whether the disruption of entrainment to weak entraining agents is due to a reduction in photic input, to disruption of components in the circadian rhythm generator, or both remains to be determined.

The relationship between the avian pineal and the SCN is not yet clear. Given the currently available information, there are several testable hypotheses that account for the behavior of sparrows bearing SCN lesions.

(1) The suprachiasmatic nuclei could be components of the output pathway between a circadian pacemaker located elsewhere and the overt locomotor rhythm that we measure. On this hypothesis, arrhythmicity in SCNlesioned birds is the result of uncoupling the pacemaker from its output by interrupting this pathway. If, as our data suggest, SCN-lesioned sparrows are capable of synchronization to light cycles, a damped oscillator with photic input must remain in the output pathway distal to the SCN. Specifically, the SCN could be target organs for a circadian pacemaker located in the pineal. The pineal is hormonally coupled on its output side to the rest of the circadian system (Menaker and Zimmerman, 1976) and the coupling hormone may be melatonin (Turek et al., 1976; Gwinner and Benzinger, 1978), an indoleamine of pineal origin. Melatonin exhibits a circadian rhythm in both pineal content and serum levels (Ralph et al., 1974). In the rat, there is evidence for the accumulation in the SCN of indoleamines that crossreact with antibodies to melatonin (Bubenik et al., 1976). The specific form of this hypothesis predicts that a selfsustained rhythm of melatonin production would persist in sparrows bearing SCN lesions.

(2) The SCN could play a permissive role and be necessary for sustaining pineal rhythmicity in vivo in constant conditions. Although neural connections of the pineal do not appear to be necessary for sustaining circadian locomotor rhythmicity in sparrows (Zimmerman and Menaker, 1975), hormonal pathways to the pineal involving the SCN cannot be excluded. This hypothesis predicts that the pineal would not be capable of oscillation in SCN-lesioned sparrows. This hypothesis appears unlikely, however, because chicken pineals can oscillate when isolated in vitro (Kasal et al., 1979; Takahashi et al., 1980).

(3) The SCN and the pineal gland may interact and function together as a complex pacemaker. In this case, neither structure alone would be able to sustain the normal overt rhythmicity of the bird if the other was absent, and neither the SCN nor the pineal would be independently capable of self-sustained oscillation. ${ }^{4} \mathrm{Or}$ gan culture experiments suggest that the chicken pineal behaves as a damped oscillator when isolated in vitro (Takahashi et al., 1980). Pinealectomized sparrows express damped locomotor rhythms, raising the possibility that the sparrow SCN behaves as a damped oscillator. Thus, an intriguing form of this hypothesis is that the self-sustained oscillation generated by the intact bird results from the interaction of two damped oscillatory components.

If we assume that the pineal and the SCN behave as damped oscillators, then the results from the pineal "phase transplant" experiments of Zimmerman and Menaker (1979) can be interpreted in the following way. Pinealectomized birds appear arrhythmic as a result of damping or uncoupling of a population of oscillators located in the SCN. Normally, these oscillators are entrained by a rhythmic hormonal signal, perhaps mela-

${ }^{4}$ This hypothesis was proposed by Dr. Felix Strumwasser in Suda et al. (1979), p. 441. 
tonin, from the pineal. When the pineal is transplanted or isolated in organ culture, it behaves as a damped oscillator which conserves the phase of the rhythm of the donor for at least several cycles. The damped hormonal signal from the transplanted pineal is sufficient to synchronize the population of SCN oscillators and to initiate a coherent rhythm which emerges with a phase related to the donor. The newly initiated rhythm in the SCN then could generate a rhythmic signal that feeds back on the pineal and maintains the amplitude of the pineal oscillation. The feedback signal could be transmitted by the sympathetic fibers that innervate both the pineal and the iris. Because pineals become supersensitive when neurally isolated (Deguchi and Axelrod, 1973), rapid reinnervation of the transplant may not be necessary if transmitter substances are released from terminals in the iris. Alternatively, the feedback signal could be hormonal. In either case, the observed rapid establishment of rhythmicity in transplant recipients could easily occur. In this way, the SCN and the pineal could generate a self-sustained behavioral oscillation through mutual entrainment. If correct, this hypothesis implies that the circadian properties of the system are to be accounted for at, but not below, the organismal level.

\section{References}

Bendat, J. S., and A. G. Piersol (1971) Random Data: Analysis and Measurement Procedures, John Wiley \& Sons, New York.

Blackman, R., and J. Tukey (1958) The Measurement of Power Spectra, Dover, New York.

Bons, N. (1976) Retino-hypothalamic pathway in the duck (Anas platyrhynchos). Cell Tissue Res. 168: 343-360.

Bouillé, C., S. Herbuté, and J. D. Baylé (1973) Modulatory influences of the central nervous structures in pituitary-adrenocortical activity: Effect of chronic deafferentation of the hypothalamus in the pigeon. J. Physiol. (Paris) 66: 437-446.

Bouillé, C., S. Herbuté, and J. D. Baylé (1975) Effects of hypothalamic deafferentation on basal and stress-induced adrenocortical activity in the pigeon. J. Endocrinol. 66: 413-419.

Brown-Grant, K., and G. Raisman (1977) Abnormalities in reproductive function associated with the destruction of the suprachiasmatic nuclei in female rats. Proc. $R$. Soc. Lond. (Biol.) 198: 279-296.

Bubenik, G. A., G. M. Brown, and L. J. Grota (1976) Differential localization of $N$-acetylated indolealkylamines in CNS and the Harderian gland using immunohistology. Brain Res. 118: $417-427$

Cooley, J. W., and J. W. Tukey (1965) An algorithm for machine calculation of complex Fourier series. Math. Comput. 19: 297-301.

Crosby, E. C., and M. J. C. Showers (1969) Comparative anatomy of the preoptic and hypothalamic areas. In The Hypothalamus, W. Haymaker, E. Anderson, and W. J. H. Nauta, eds., pp. 61-135, Charles C Thomas, Springfield, IL.

Crosby, E. C., and R. T. Woodburne (1940) The comparative anatomy of the preoptic area and the hypothalamus. Proc. Assoc. Res. Nerv. Ment. Dis. 20: 52-169.

Davies, D. T. (1980) The neuroendocrine control of gonadotropin release in the Japanese quail. III. The role of the tuberal and anterior hypothalamus in the control of ovarian development and ovulation. Proc. R. Soc. Lond. (Biol.) 206: 421-437.

Deguchi, T., and J. Axelrod (1973) Supersensitivity and subsensitivity of the $\beta$-adrenergic receptor in pineal gland regulated by catecholamine transmitter. Proc. Natl. Acad. Sci. U. S. A 70: 2411-2414.

Ebihara, S., and H. Kawamura (1981) The role of the pineal organ and the suprachiasmatic nucleus in the control of circadian locomotor rhythms in the Java sparrow, Padda oryzivora. J. Comp. Physiol. 141: 207-214.

Eichler, V. B., and R. Y. Moore (1974) The primary and accessory optic systems in the golden hamster, Mesocricetus auratus. Acta Anat. (Basel) 89: 359-371.

Gaston, S. (1971) The influence of the pineal organ on the circadian rhythm in birds. In Biochronometry, M. Menaker, ed., pp. 541-548, National Academy of Sciences, Washington, DC.

Gaston, S., and M. Menaker (1968) Pineal function: The biological clock in the sparrow? Science 160: 1125-1127.

Gwinner, E., and I. Benzinger (1978) Synchronization of a circadian rhythm in pinealectomized European starlings by daily injections of melatonin. J. Comp. Physiol. 127: 209-214

Harris, F. J. (1978) On the use of windows for harmonic analysis with the discrete Fourier transform. Proc. IEEE 66: 51-83.

Hartwig, H. -G. (1974) Electron microscopic evidence for a retino-hypothalamic projection to the suprachiasmatic nuclei of Passer domesticus. Cell Tissue Res. 153: 89-99.

Hendrickson, A. E., N. Wagoner, and W. M. Cowan (1972) Autoradiographic and electron microscope study of retinohypothalamic connections. Z. Zellforsch. Mikrosk. Anat. 125: $1-26$.

Ibuka, N., S. I. T. Inouye, and H. Kawamura (1977) Analysis of sleep-wakefulness rhythms in male rats after suprachiasmatic nucleus lesions and ocular enucleation. Brain Res. 122: 33-48.

Inouye, S. T., and H. Kawamura (1979) Persistence of circadian rhythmicity in a mammalian hypothalamic "island" containing the suprachiasmatic nucleus. Proc. Natl. Acad. Sci U. S. A. 76: 5962-5966.

Kasal, C., M. Menaker, and R. Perez-Polo (1979) Circadian clock in culture: $N$-Acetyltransferase activity of chick pineal glands oscillates in vitro. Science 203: 656-658.

Kluver, H., and E. Barrera (1953) A method for the combined staining of cells and fibers in the nervous system. J. Neuropathol. Exp. Neurol. 12: 400-403.

McMillan, J. P. (1972) Pinealectomy abolishes the circadian rhythm of migratory restlessness. J. Comp. Physiol. 79: 105-112.

McMillan, J. P., H. C. Keatts, and M. Menaker (1975) On the role of eyes and brain photoreceptors in the sparrow: Entrainment to light cycles. J. Comp. Physiol. 102: 251-256.

Meier, R. E. (1973) Autoradiographic evidence for a direct retinohypothalamic projection in the avian brain. Brain Res. 53: 417-421.

Menaker, M., and A. Eskin (1966) Entrainment of circadian rhythms by sound in Passer domesticus. Science 154: $1579-1581$.

Menaker, M., and H. Underwood (1976) Extraretinal photoreception in birds. Photochem. Photobiol. 23: 299-306.

Menaker, M., and N. Zimmerman (1976) Role of the pineal in the circadian system of birds. Am. Zool. 16: 45-55.

Menaker, M., J. S. Takahashi, and A. Eskin (1978) The physiology of circadian pacemakers. Annu. Rev. Physiol. 40: 501-526.

Moore, R. Y. (1978) Central neural control of circadian rhythms. In Frontiers in Neuroendocrinology, W. F. Ganong and L. Martin, eds., Vol. 5., pp. 185-206, Raven Press, New York.

Moore, R. Y., and V. B. Eichler (1972) Loss of a circadian adrenal corticosterone rhythm following suprachiasmatic lesions in the rat. Brain Res. 42: 201-206.

Moore, R. Y., and D. C. Klein (1974) Visual pathways and the central neural control of a circadian rhythm in pineal serotonin $N$-acetyltransferase activity. Brain Res. 71: 17-33.

Moore, R. Y., and N. J. Lenn (1972) A retinohypothalamic 
projection in the rat. J. Comp. Neurol. 146: 1-14.

Otnes, R. K., and L. Enochson (1978) Applied Time Series Analysis, Vol. 1., John Wiley \& Sons, New York.

Ralph, C. L. (1959) Some effects of hypothalamic lesions on gonadotropin release in the hen. Anat. Rec. 134: 411-431.

Ralph, C. L., R. W. Pelham, S. E. MacBride, and D. P. Reilly (1974) Persistent rhythms of pineal and serum melatonin in cockerels in continuous darkness. J. Endocrinol. 63: 319-324.

Rusak, B. (1977) The role of the suprachiasmatic nuclei in the generation of circadian rhythms in the golden hamster, $M e$ socricetus auratus. J. Comp. Physiol. 118: 145-164.

Rusak, B., and I. Zucker (1979) Neural regulation of circadian rhythms. Physiol. Rev. 59: 449-526.

Schwartz, W. J., L. C. Davidsen, and C. B. Smith (1980) In vivo metabolic activity of a putative circadian oscillator, the rat suprachiasmatic nucleus. J. Comp. Neurol. 189: 157-167.

Simpson, S. M., and B. K. Follett (1980) Investigations on the possible roles of the pineal and anterior hypothalamus in regulating circadian activity rhythms in Japanese quail. In Acta XVII Congressus Internationalis Ornithologici, R. Nohring, ed., Vol. I., pp. 435-438, Deutschen OrnithologenGesellschaft, Berlin.

Singleton, R. C. (1969) An algorithm for computing the mixed radix fast Fourier transform. IEEE Trans. Aud. Electroacoustics 17: 93-103.

Stephan, F. K., and I. Zucker (1972) Circadian rhythms in drinking behavior and locomotor activity of rats are eliminated by hypothalamic lesions. Proc. Natl. Acad. Sci. U. S. A. 69 : 1583-1586.
Stetson, M. H., and M. Watson-Whitmyre (1976) Nucleus suprachiasmaticus: The biological clock in the hamster? Science 191: 197-199.

Suda, M., O. Hayaishi, and N. Nakagawa (1979) Biological Rhythms and Their Central Mechanism, Elsevier/NorthHolland, Amsterdam.

Takahashi, J. S., and M. Menaker (1979a) Physiology of avian circadian pacemakers. Fed. Proc. 38: 2583-2588.

Takahashi, J. S., and M. Menaker (1979b) Brain mechanisms in avian circadian systems. In Biological Rhythms and Their Central Mechanism, M. Suda, O. Hayaishi, and H. Nakagawa, eds., pp. 95-109, Elsevier/North-Holland, Amsterdam.

Takahashi, J. S., and M. Menaker (1982) Entrainment of the circadian system of the house sparrow: A population of oscillators in pinealectomized birds. J. Comp. Physiol. 146: 245-253.

Takahashi, J. S., H. Hamm, and M. Menaker (1980) Circadian rhythms of melatonin release from individual superfused chicken pineal glands in vitro. Proc. Natl. Acad. Sci. U. S. A. 77: $2319-2322$.

Turek, F. W., J. P. McMillan, and M. Menaker (1976) Melatonin: Effects on the circadian locomotor rhythm of sparrows. Science 194: 1441-1443.

Zimmerman, N. H., and M. Menaker (1975) Neural connections of sparrow pineal: Role in circadian control of activity. Science 190: 477-479.

Zimmerman, N. H., and M. Menaker (1979) The pineal: A pacemaker within the circadian system of the house sparrow. Proc. Natl. Acad. Sci. U. S. A. 76: 999-1003. 